\title{
The measured equation of invariance and its application to transmission line modelling
}

\author{
Rafael Pous(1), Mark D. Prouty(2), and \\ Kenneth K. Mei(2)
}

(1) Universitat Politècnica de Catalunya, TSC Department, P.O. Box 30.002, 08080 Barcelona, Spain, Ph: (34 3) 4017199 , Fax:

(343) 401 7232, e-mail:pous@voltor.upc.es

(2) University of California at Berkeley, EECS Department

\section{Abstract}

The Measured Equation of Invariance (MEI) is a geometrydependent Finite Difference equation that can be used to terminate a mesh extremely close to the object of interest. The mesh can be terminated much closer than what absorbing boundary conditions would allow, but still keeping the locality of the equations. In this paper, this new concept is applied to the numerical simulation of transmission lines and their discontinuities.

\section{Introduction}

The standard Finite Difference (FD) and Finite Element (FE) methods must satisfy all the boundary conditions numerically. This is especially difficult in open-boundary problems where the mesh must extend well beyond the region of interest just to be able to apply the absorbing boundary conditions. Integral equation methods, such as the Method of Moments (MOM) or Boundary Element Method (BEM) use the Green function, which already incorporates the proper boundary conditions at infinity, but result in full associated matrices that are costly to store and invert. By using the MEI, which incorporates the information contained in the Green function to the FD or FE method, the mesh can be drastically reduced, but the matrix is kept sparse, combining the advantages of the two types of methods.

\section{The Measured Equation of Invariance}

We postulate the existence of a FD-type equation of the form $[1,2]$

$$
\phi\left(\bar{r}_{0}\right)+\sum_{i=1}^{N-1} c_{i} \cdot \phi\left(\bar{r}_{i}\right)=0
$$

relating the point $\overline{\mathrm{r}}_{0}$ and its $\mathrm{N}-1$ neighbors, which is different from the standard FD equation and is

- Dependent on the position in space.

- Dependent on the geometry of the object.

- Independent of the excitation of the problem.

The N-1 coefficients in Eqn. 1 are chosen so that they satisfy $\mathrm{N}-1$ solutions to the problem $\phi^{\mathrm{k}}(\overline{\mathrm{r}})$, which we call measuring functions. In other words, the coefficients in 1 are found using N-1 known solutions to the problem. Since the coefficients are invariant to the excitation, any set of linearly independent solutions will yield the same coefficients.

To find $\mathrm{N}-1$ solutions is in principle as difficult as solving the problem at hand, but since they can be chosen arbitrarily, we can construct $\mathrm{N}-1$ solutions from $\mathrm{N}-1$ sources and the Green function of the problem in the following way

$$
\phi^{k}(\bar{r})=\int_{C} \sigma^{k}\left(\bar{r}^{\prime}\right) \cdot G\left(\bar{r} \mid \bar{r}^{\prime}\right) \cdot d \bar{r}^{\prime}
$$

We call this sources $\sigma^{\mathbf{k}}$, which can be chosen arbitrarily, metrons.

The coefficients are the ones satisfying the $\mathrm{N}-1$ equations

$$
\sum_{i=1}^{N-1} c_{i} \cdot \phi^{k}\left(\bar{r}_{i}\right) \quad k=1 \ldots N-1
$$

Alternatively, we may use more than $\mathrm{N}-1$ metrons, but then we need to find the coefficients that are a least square fit to the overdetermined system of equations.

\section{TEM Transmission Lines}

First we will consider a TEM transmission line such as the one shown in Fig. 1. In a standard FD solution, the whole cross section of the transmission line would have to be covered by the mesh, adding a lot of complexity to the generation of the mesh and the inversion of the matrix. Since part of the geometry is canonical (i.e. the outer conductor), it can be incorporated in the Green function. The standard FD equations can be used for all the points in the interior of the mesh and on the metal. For the points on the exterior of the mesh, Eqn. 1 must be used. The 5 closest neighbors are used $(\mathrm{N}=6)$, the 5 metrons are

$$
\begin{aligned}
\sigma^{1}\left(l^{\prime}\right) & =1 \\
\sigma^{2}\left(l^{\prime}\right) & =\cos 2 \pi \frac{l^{\prime}}{L} \\
\sigma^{2}\left(l^{\prime}\right) & =\sin 2 \pi \frac{l^{\prime}}{L} \\
\sigma^{2}\left(l^{\prime}\right) & =\cos 4 \pi \frac{l^{\prime}}{L} \\
\sigma^{2}\left(l^{\prime}\right) & =\sin 4 \pi \frac{l^{\prime}}{L}
\end{aligned}
$$

and the Green function is the Green function for a source inside a parallel plate waveguide [3]. The boundary condition on the object is $\phi=1 \mathrm{~V}$. Figure 1 also shows the characteristic impedance of the line for different values of $w$, compared with the MOM results.

The MEI can also be used to solve multiconductor problems. The mesh must enclose each of the conductors, but it must be connected. In this type of cases an umbilical must be used to connect the mesh around each conductor. Figure 2 shows the mesh used to calculate the coupling between two air-microstrip lines placed on top of each other, and the values of the capacitance matrix elements, compared with the Spectral Domain approach. Note that a mesh is drawn around each line, and two umbilical meshes are used to connect them. The MEI equations are used for every point of the mesh not in the interior, on the metal, or on the plane of symmetry. The Green function for this problem is

$$
G\left(\bar{r} \mid \bar{r}^{\prime}\right)=\frac{-1}{4 \pi \epsilon_{o}} \ln \frac{\left(x-x^{\prime}\right)^{2}+\left(y-y^{\prime}\right)^{2}}{\left(x-x^{\prime}\right)^{2}+\left(y+y^{\prime}\right)^{2}}
$$

if the ground plane is at $\mathrm{y}=0$.

Figure 3 shows the results of using a quasi-static Green function to calculate the characteristic impedance of a microstrip line. The MEI can also be used to find a fullwave solution if a full-wave Green function is used instead.

\section{Discontinuities in Planar Transmission Lines}

For the study of discontinuities in a transmission line, a threedimensional formulation is necessary. For the special case of planar structures (microstrip, stripline, coplanar, etc...), the currents are confined to two dimensions (xy), and the formulation is greatly simplified by using as the unknowns the two components of the vector potential $\vec{A}$ in the direction of the currents. We will only consider the case with no dielectric substrate $\left(\varepsilon_{\mathrm{r}}=1\right)$.

The vector potential satisfies the wave equation, which can be used for points interior to the mesh. On the metal, the boundary conditions must be applied $\vec{E}_{\tan }$. The expression for the scattered tangential electric field is 


$$
\begin{aligned}
& E_{x}^{s}=\frac{-j \omega}{k_{o}^{2}}\left[\left(\frac{\partial^{2}}{\partial x^{2}}+k_{o}^{2}\right) A_{x}+\frac{\partial^{2}}{\partial x \partial y} A_{y}\right] \\
& E_{y}^{s}=\frac{-j \omega}{k_{o}^{2}}\left[\left(\frac{\partial^{2}}{\partial y^{2}}+k_{o}^{2}\right) A_{y}+\frac{\partial^{2}}{\partial x \partial y} A_{y}\right]
\end{aligned}
$$

Using the fact that $\mathrm{Hz}$ vanishes on a conductor in the xy plane, we can write

$$
\begin{aligned}
& E_{x}^{s}=\frac{-j \omega}{k_{o}^{2}}\left(\frac{\partial^{2}}{\partial x^{2}}+\frac{\partial^{2}}{\partial y^{2}}+k_{o}^{2}\right) A_{x}+\frac{j \omega}{k_{o}^{2}} \frac{\partial}{\partial y} H_{z}^{i} \\
& E_{y}^{s}=\frac{-j \omega}{k_{o}^{2}}\left(\frac{\partial^{2}}{\partial x^{2}}+\frac{\partial^{2}}{\partial y^{2}}+k_{o}^{2}\right) A_{y}-\frac{j \omega}{k_{o}^{2}} \frac{\partial}{\partial x} H_{z}^{i}
\end{aligned}
$$

Depending on the local geometry, one of the two expression may be used to enforce the boundary condition on the metal.

Figure 4 shows the mesh used for this type of calculation. To terminate the mesh we use the MEI. To derive it, we need the Green function and a set of metrons. In this type of problems,

$$
\overline{\bar{G}}\left(\bar{r} \mid \bar{r}^{\prime}\right)=\left[\begin{array}{ll}
1 & 0 \\
0 & 1
\end{array}\right] \cdot \frac{e^{-j k_{o}\left|\bar{r}-\bar{r}^{\prime}\right|}}{4 \pi\left|\bar{r}-\bar{r}^{\prime}\right|}
$$

delta source metrons have been found to yield good results. With these metrons, the measuring functions are directly the Green function with sources at different points on the transmission lines. We used one such metron at each mesh point on the metal.
Figure 5 shows the currents near a step-gap discontinuity. Figure 6 shows the longitudinal current near a double step discontinuity.

\section{Conclusion}

A new concept in numerical simulation has been applied to the solution of a number of transmission line problems. It is shown that the MEI, which has extensively been tested in scattering problems, can also be used in guided wave cases. In fact, the only difference between the solution of a transmission line and a $2 \mathrm{D}$ scattering problem is the Green function in 2.

\section{References}

[1] K.K. Mei, R. Pous, Z. Chen, Y.W. Liu, and M.D. Prouty, "The Measured Equation of Invariance: a new concept in field computation," IEEE Trans. Antennas and Propagat., submitted for publication.

[2] R. Pous, "The Measured Equation of Invariance: a new concept in field computation," Ph.D. thesis, Univ. of California, Berkeley, 1992.

[3] R.E. Collin, Field Theory of Guided Waves, IEEE Press, New York, 1990

[4] O.M. Ramahi, A. Khebir, and R. Mittra, "Numerically derived absorbing boundary condition for the solution of open region scattering problems," IEEE Trans. Antennas and Propagat., vol. 38, pp. 1551-1558, 0ct. 1990.
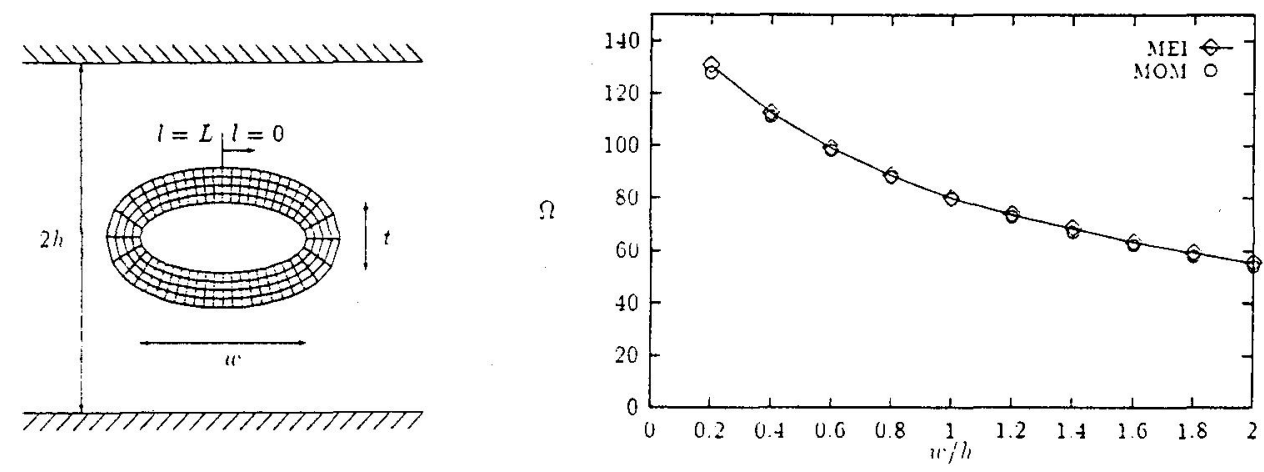

Figure 1: Cross section of a TEM transmission line with the FD mesh used in the solution, and its characteristic impedance for different values of $w(t=0.4 h)$.

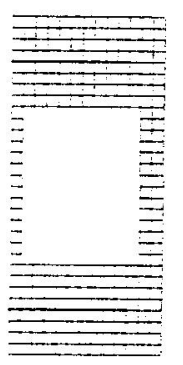

\begin{tabular}{|l|l|l|l|}
\hline Method & $\mathrm{C} 1$ & $\mathrm{C} 2$ & $\mathrm{Cm}$ \\
\hline MEI & 2.574 & 3.783 & 1.064 \\
\hline SDA & 2.616 & 3.755 & 1.056 \\
\hline
\end{tabular}

Figure 2: Mesh used to calculate the coupling between two air-microstrip lines, and the capacitance matrix elements compared with the Spectral Domain approach (drawn to scale). 

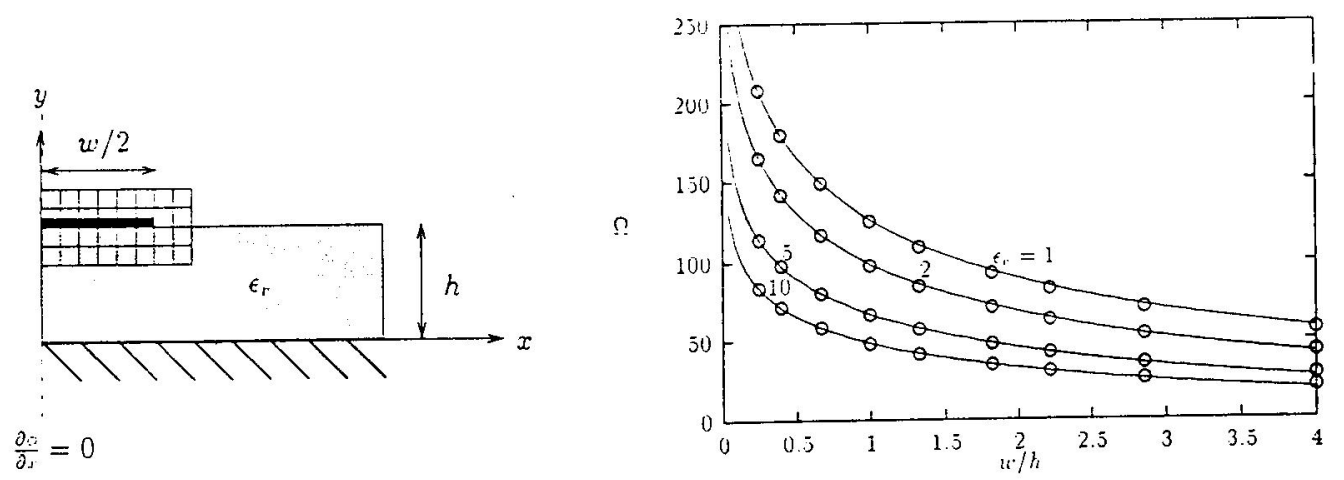

Figure 3: Characteristic impedance of a microstrip line calculated with a quasi-static MEI (dots) compared with Wheeler's formula (line).

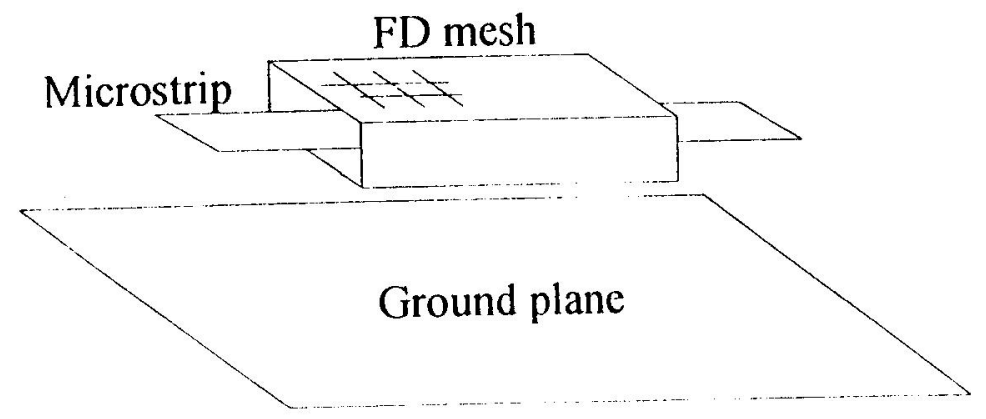

Figure 4: Mesh used around a microstrip discontinuity.

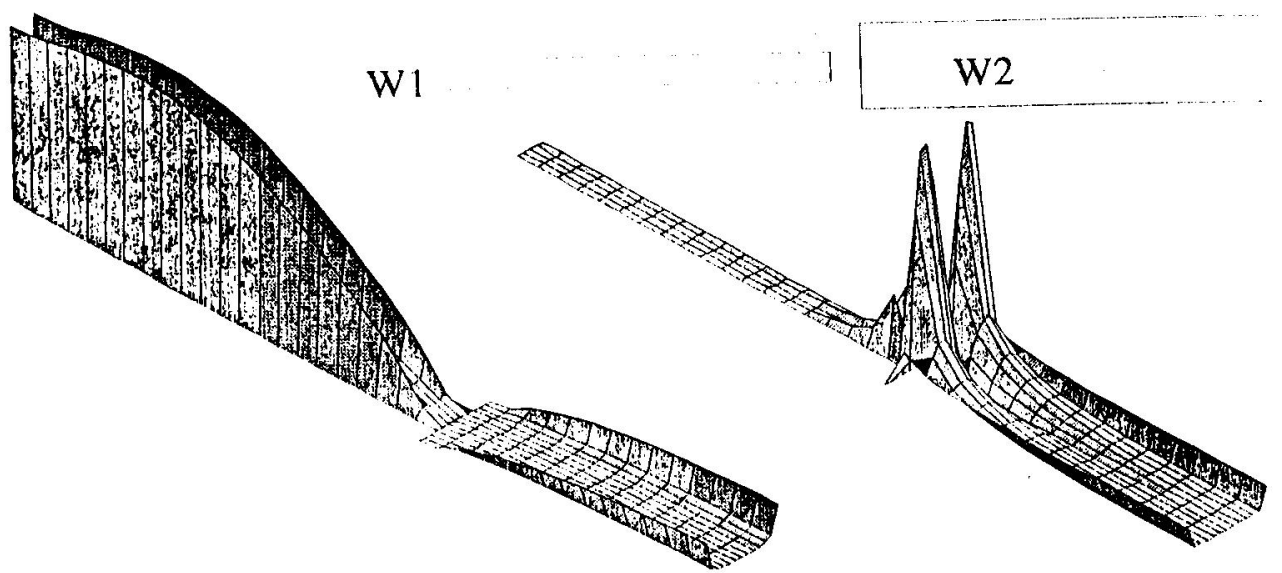

Figure 5: Currents on a step gap structure. The magnitude of the longitudinal component is shown on the left, and the transverse component (magnified 20 times) is shown on the right $(W 1=3 W 2$, gap width $=W 1, \lambda=15 W 1, H=2.5 W 1)$.

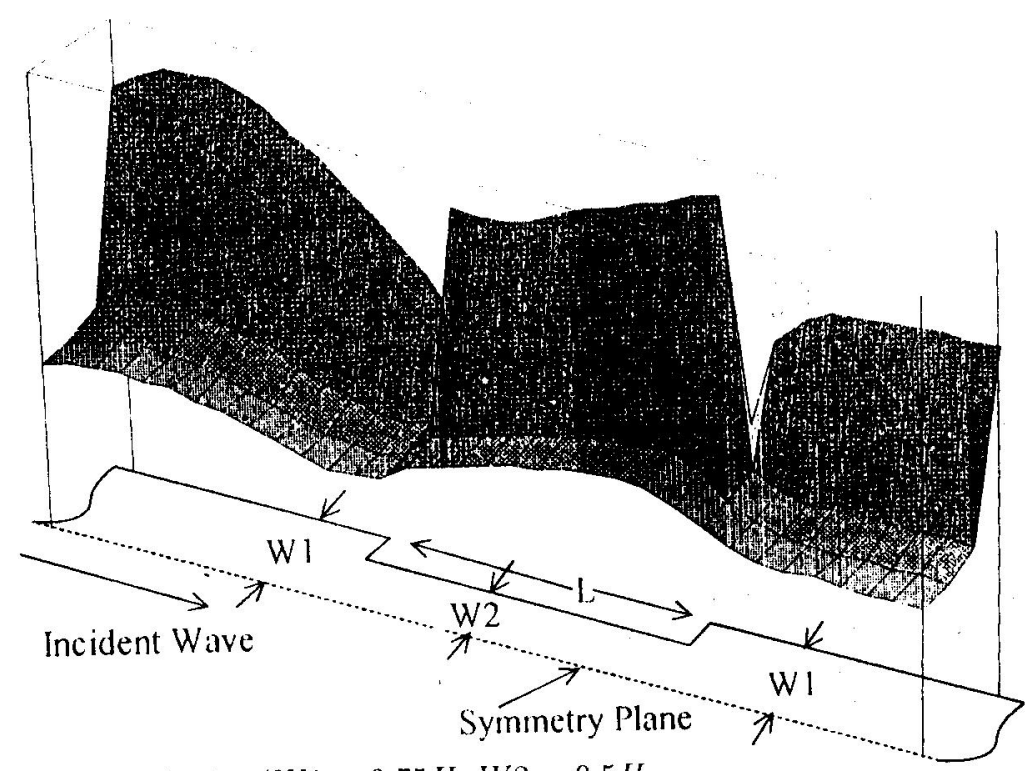

Figure 6: Longitudinal current on a double step discontinuity $(W 1=0.75 \mathrm{H}, \mathrm{W} 2=0.5 \mathrm{H}$, $L=2.75 H, H$ is height above the ground plane, $L=\lambda / 4$ ). 